\title{
Ant Colony System-Based Algorithm for Optimal Multi-stage Planning of Distribution Transformer Sizing
}

\author{
Eleftherios I. Amoiralis ${ }^{1}$, Pavlos S. Georgilakis ${ }^{1}$, Marina A. Tsili ${ }^{2}$, \\ and Antonios G. Kladas ${ }^{2}$ \\ ${ }^{1}$ Department of Production Engineering and Management, Technical University of Crete, \\ University Campus, Chania, Greece \\ eamir@tee.gr, pgeorg@dpem.tuc.gr \\ ${ }^{2}$ Faculty of Electrical \& Computer Engineering, National Technical University of Athens \\ University Campus, Athens, Greece \\ \{mtsili, kladasel\}@eentral.ntua.gr
}

\begin{abstract}
This paper proposes a stochastic optimization method, based on ant colony optimization, for the optimal choice of transformer sizes to be installed in a distribution network. This method is properly introduced to the solution of the optimal transformer sizing problem, taking into account the constraints imposed by the load the transformer serves throughout its life time and the possible transformer thermal overloading. The possibility to upgrade the transformer size one or more times throughout the study period results to different sizing paths, and ant colony optimization is applied in order to determine the least cost path, taking into account the transformer capital cost as well as the energy loss cost during the study period. The results of the proposed method demonstrate the benefits of its application in the distribution network planning.
\end{abstract}

Keywords: Transformers; Optimal Transformer Sizing; Ant Colony Optimization; Thermal Loading; Energy Loss Cost; Distribution Network Planning.

\section{Introduction}

The objective of the optimal transformer sizing problem in a multi-year planning period is to select the transformer sizes (i.e., rated capacities) and the years of transformer installation so as to serve a distribution substation load at the minimum total cost (i.e., sum of transformer purchasing cost plus transformer energy loss cost). Deterministic optimization methods may be used for the solution of this problem, such as dynamic programming [1] or integer programming [2]. However, the wide spectrum of transformer sizes and various load types involved in the electric utility distribution system make the transformer sizing a difficult combinatorial optimization problem, since the space of solutions is huge. That is why stochastic optimization methods may prove to provide more robust solutions.

In this paper, the Optimal Transformer Sizing (OTS) problem is solved by means of the heuristic Ant System method using the Elitist strategy, called Elitist Ant System (EAS). EAS belongs to the family of Ant Colony Optimization (ACO) algorithms. 
Dorigo has proposed the EAS in [3]. The EAS is a biologically inspired meta-heuristics method in which a colony of artificial ants cooperates in finding good solutions to difficult discrete optimization problems, such as the OTS problem. Cooperation is the key design component of ACO algorithms, i.e. allocation of the computational resources to a set of relatively simple agents (artificial ants) that communicate indirectly by stigmergy (by indirect communication mediated by the environment). In other words, a set of artificial ants cooperate in dealing with a problem by exchanging information via pheromones deposited on a graph. In the literature, ACO algorithms have been applied to solve a variety of well-known combinatorial optimization problems, such as routing [4], scheduling [5], and subset [6] problems. More details on ACO application in the solution of other problems are described in [7].

EAS was introduced in the solution of the OTS problem in case of one (threephase, oil-immersed) distribution transformer with constant economic factors in [8], whereas this paper extends the use of EAS, taking into account all details of the economic analysis, such as the inflation rate that influences the energy loss cost and the transformer investment as well as the installation and depreciation cost in a real distribution network, constituting an efficient methodology for transformer planning. The OTS problem is solved as a constrained optimization problem.

\section{Overview of the Proposed Method}

The OTS problem consists in finding the proper capacities of transformers to be installed in a distribution network so that the overall installation and energy loss cost over the study period is minimized and the peak loading condition is met [1]. The proposed solution to the OTS problem is described in Fig. 1.
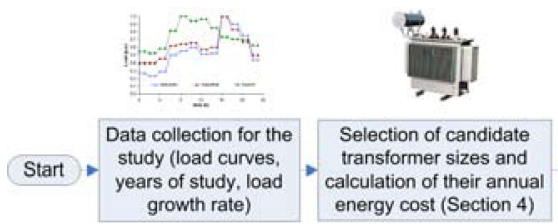

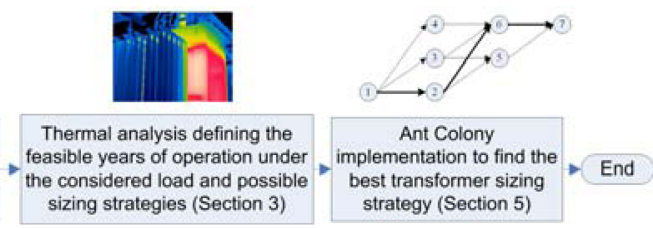

Fig. 1. Flowchart illustrating the main steps of the proposed method

\section{Calculation of Transformer Thermal Loading}

The transformer thermal calculation is implemented according to the guidelines imposed by the IEEE Standard C57.91-1995 (R2002), [9]. The transformer top-oil rise over ambient temperature and winding hottest spot temperature are calculated during each interval of the considered load cycle, taking properly into account its constructional characteristics as well as the no-load and load loss. For the study of the present paper, a maximum hot-spot temperature of $120^{\circ} \mathrm{C}$ has been chosen, based on the relative aging rate of the insulation in the transformer. For the determination of loading limits, the calculation of the hottest spot temperature is repeated for each year of the study period, at an hourly basis, according to the daily load curve. To remain on the safe side, 
the peak load curve of the considered year is used in the calculations. The yearly load growth rate $s$ is taken into consideration for the derivation of the per unit load $K_{t}^{k}$ of hour $t$ at year $k$ of the study based on the per unit load $K_{t}^{0}$ of hour $t$ at year 0 :

$$
K_{t}^{k}=K_{t}^{0} \cdot(1+s)^{k} .
$$

Fig. 2 shows the winding hottest spot temperature variation for six distribution transformers of rated capacity 160, 250,300, 400, 500 and $630 \mathrm{kVA}$, serving a residential load of $398 \mathrm{kVA}$ peak value at the 14th year of the study period (this load has $230 \mathrm{kVA}$ peak value at the beginning of the study and $4 \%$ annual load growth). As can be observed from Fig. 2, the 160, 250 and $300 \mathrm{kVA}$ transformers overcome the hottest spot limit of $120^{\circ} \mathrm{C}$ so they are not suitable to serve the load at the $14^{\text {th }}$ year of the study period.

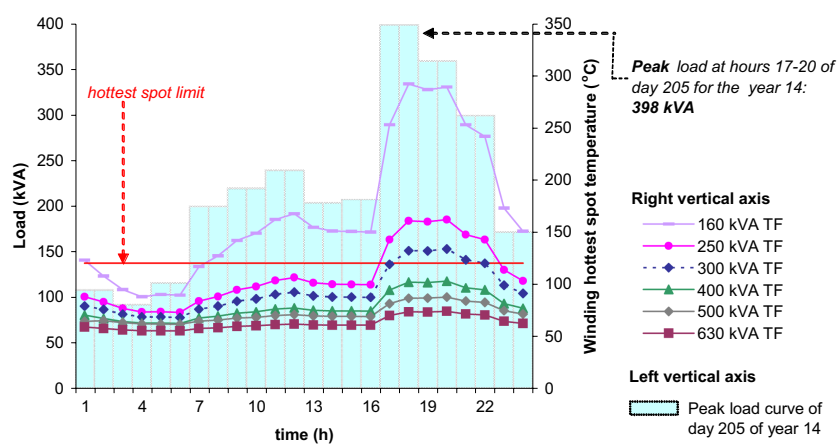

Fig. 2. Winding hottest spot temperature variation of six transformer (TF) ratings

\section{Calculation of Transformer Energy Loss Cost}

The calculation of annual transformer energy loss cost of the potential sizing paths is realized with the use of the energy corresponding to the transformer no-load loss $(N L L) E_{N L L}$ (in $\mathrm{kWh}$ ) for each year of operation and the energy corresponding to the load loss $(L L), E_{L L}^{k}$ (in $\mathrm{kWh}$ ) for each $k$-th year of operation. These energies are calculated according to (2) and (3), respectively:

$$
E_{N L L}=N L L \cdot H P Y \quad(2), \quad E_{L L}^{k}=L L \cdot\left[l_{f} \cdot \frac{S_{\max , 0}^{l}}{S_{n o m}^{l}}(1+s)^{k}\right]^{2} \cdot H P Y
$$

where $S_{\text {max }, 0}^{l}$ is the initial peak load of the substation load type $l$ (in kVA), $S_{\text {nom }}^{l}$ is the nominal power of the transformer that serves load type $l$ (in $\mathrm{kVA}$ ), $H P Y$ is the number of hours per year, equal to 8760 , and $l_{f}$ is the load factor, i.e. the mean transformer loading over its lifetime (derived from the load curve of each consumer type served by the considered substation). The cost of total energy corresponding to the transformer $N L L$ for each $k$-th year $C_{N L L}^{k}$ (in $€$ ) and the cost of energy corresponding to the transformer $L L$ for each $k$-th year $C_{L L}^{k}$ (in $€$ ) are calculated as follows: 


$$
C_{N L L}^{k}=E_{N L L} \cdot C_{Y E C}^{k} \quad(4), \quad C_{L L}^{k}=E_{L L}^{k} \cdot C Y E C^{k}
$$

where $C Y E C^{k}$ denotes the present value of the energy cost (in $€ / \mathrm{kWh}$ ) at the $k$-th year. Finally, the total cost of the transformer energy loss $C_{L}^{k}$ for the $k$-th year is given by:

$$
C_{L}^{k}=C_{N L L}^{k}+C_{L L}^{k}
$$

\section{Elitist Ant System Method}

\subsection{Mechanism of EAS Algorithm}

The EAS is an evolutionary computation optimization method based on ants' collective problem solving ability. This global stochastic search method is inspired by the ability of a colony of ants to identify the shortest route between the nest and a food source, without using visual cues.

The operation mode of EAS algorithm is as follows: the artificial ants of the colony move, concurrently and asynchronously, through adjacent states of a problem, which can be represented in the form of a weighted graph. This movement is made according to a transition rule, called random proportional rule, through a stochastic mechanism. When ant $k$ is in node $i$ and has so far constructed the partial solution $s^{p}$, the probability of going to node $j$ is given by:

$$
p_{i j}^{k}=\left\{\begin{array}{cl}
\frac{\tau_{i j}^{\alpha}+n_{i j}^{\beta}}{\sum_{c_{i l} \in N\left(s^{p}\right)} \tau_{i l}^{\alpha}+n_{i l}^{\beta}}, & \text { if } c_{i j} \in N\left(s^{p}\right) \\
0 & , \text { otherwise }
\end{array}\right.
$$

where $N\left(s^{p}\right)$ is the set of feasible nodes when being in node $i$, i.e. edges $(i, l)$ where $l$ is the node not yet visited by the ant $k$. The parameters $\alpha$ and $\beta$ control the relative importance of the pheromone versus the heuristic information value $\eta_{i j}$, given by:

$$
n_{i j}=\frac{1}{d_{i j}}
$$

where $d_{i j}$ is the weight of each edge.

Individual ants contribute their own knowledge to other ants in the colony by depositing pheromones, which act as chemical "markers" along the paths they traverse. Through indirect communication with other ants via foraging behavior, a colony of ants can establish the shortest path between the nest and the food source over time with a positive feedback loop known as stigmergy. As individual ants traverse a path, pheromones are deposited along the trail, altering the overall pheromone density. More trips can be made along shorter paths and the resulting increase in pheromone density attracts other ants to these paths. The main characteristic of the EAS technique is that (at each iteration) the pheromone values are updated by all the $k$ ants that have built a solution in the iteration itself. The pheromone $\tau_{i j}$, associated with the edge joining nodes $i$ and $j$, is updated as follows [3]: 


$$
\tau_{i j}=(1-\rho) \cdot \tau_{i j}+\sum_{m=1}^{k} \Delta \tau_{i j}^{k}+\varepsilon \cdot \tau_{i j}^{e l i t e}
$$

where $\rho \in(0,1]$ is the evaporation rate, $k$ is the number of ants, $\varepsilon$ is the number of elitist ants, and $\Delta \tau_{i j}^{k}$ is the quantity of pheromone laid on edge $(\mathrm{i}, \mathrm{j})$ by ant $\mathrm{k}$ :

$$
\Delta \tau_{i j}^{k}=\left\{\begin{array}{l}
\frac{Q}{L_{k}}, \text { if ant } k \text { used edge }(i, j) \text { in its tour } \\
0, \text { otherwise }
\end{array}\right.
$$

where $Q$ is a constant for pheromone update, and $L_{k}$ is the length (or the weight of the edge) of the tour constructed by ant $k$. Furthermore, shorter paths will tend to have higher pheromone densities than longer paths since pheromone density decreases over time due to evaporation [3]. This shortest path represents the global optimal solution and all the possible paths represent the feasible region of the problem.

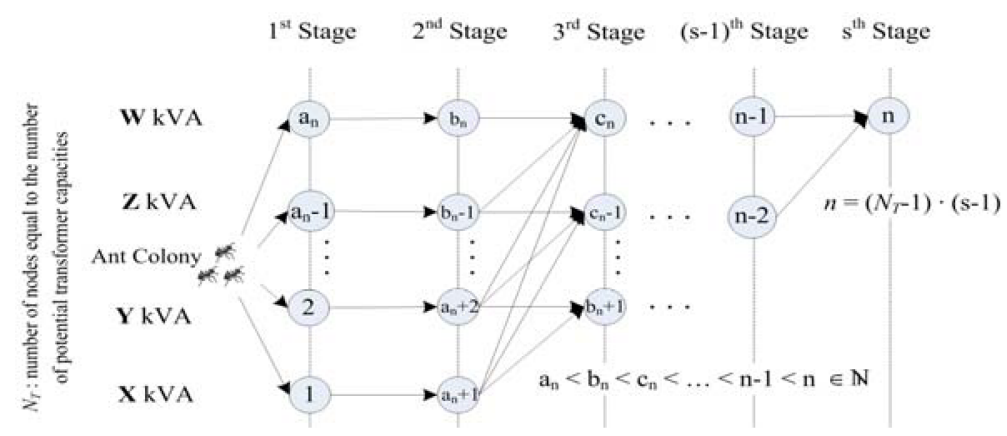

Fig. 3. The directed graph used for the OTS problem

\subsection{OTS Implementation Using the EAS Algorithm}

In this work our interest lies in finding the optimum choice of distribution transformers capacity sizing, so as to meet the load demand for all the years of the study period. To achieve so, a graph shown in Fig. 3 is constructed, representing the sizing paths.

The graph has $s$ stages and each stage indicates a time period (in years) the limits of which are defined by the need to replace one of the considered transformer sizes due to violation of its thermal loading limits. Therefore, stage $s$ has one node less in comparison with stage $(s-1)$, stage $(s-2)$ has one node less in comparison with stage $(s-1)$, etc. The first stage indicates the beginning of the study, comprising number of nodes equal to the number of potential transformer capacities $N_{T}$, while $s$ represents the end of the study period (consisting of the largest necessary rated capacity able to serve the load at the final year of the study). Symbols $X, Y, Z, W$ refer to the different rated powers $(X<Y<Z<W)$. Furthermore, the arcs between the nodes are directed from the previous stage to the next one (backward movement is not allowed) since 


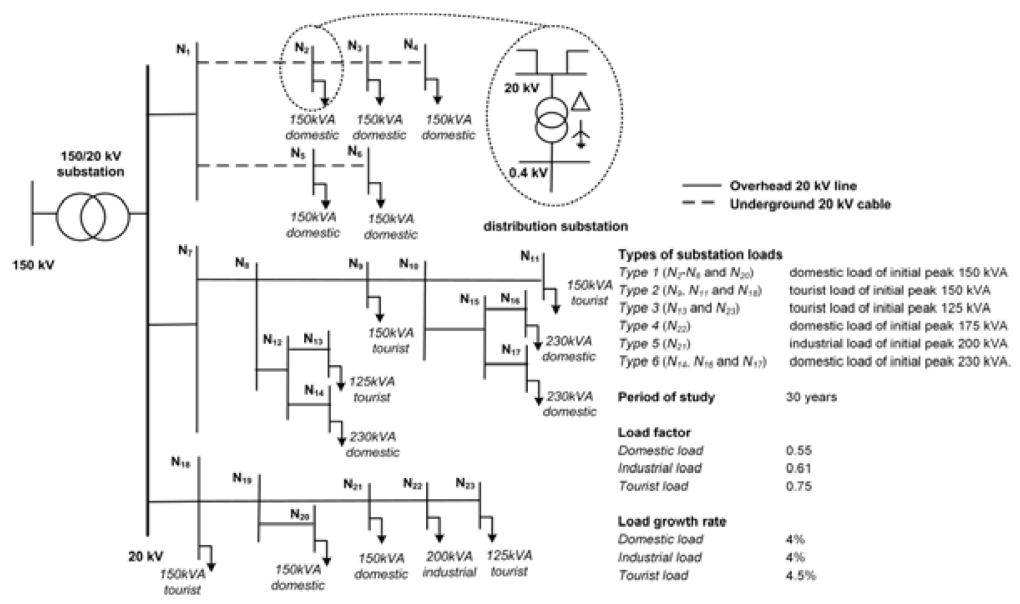

Fig. 4. Single line diagram of the examined distribution network

each stage represents a forward step in the time of the study. Nodes 1 to $a_{n}$ (Fig. 3) are designated as the source nodes corresponding to each potential transformer size and node $n$ is designated as the destination node (Fig. 3). The objective of the colony agents is to find the least-cost path between nodes that belong to 1st stage and node $n$.

\section{Results and Discussion}

The proposed method is applied for the optimal choice of the transformer sizes of a practical distribution network. Fig. 4 illustrates the single line diagram of the examined distribution network. The substation type of loads and their initial peak value (at the first year of the study period) are indicated on the diagram (Fig. 4).

Six transformer ratings are considered, namely 160, 250, 300, 400, 500 and 630 kVA. Table 1 lists their main technical characteristics and bid price. The thermal calculations illustrated in Fig. 2 were repeated for the six transformers and each year of the study, resulting to the time periods of Table 1 . The periods derived in Table 1 were used to define the stages of the graph of Fig. 3. In order to define the weight of each arc in the graph of Fig. 3, the energy loss cost calculation of each transformer for the studied period was based on the annual energy loss cost calculation described in Section 4. The energy loss cost corresponding to the transition from node $i$ ( $p$-th year of the study period) to $j$ ( $q$-th year of the study period), is computed by:

$$
C_{L}^{i \rightarrow j}=\sum_{k=p+1}^{q} C_{L}^{k}
$$

When the transition from node $i$ to $j$ corresponds to transformer size upgrade from $S_{i}$ to $S_{j}$, the installation cost $I_{i \rightarrow j}$ derives from:

$$
I_{i \rightarrow j}=B P_{S_{j}}^{p}-R_{S_{i}}^{p} \quad(12), \quad R_{S_{i}}^{p}=B P_{S_{i}} \cdot\left[\frac{(1+r)^{N}-(1+r)^{m}}{(1+r)^{N}-1}\right]
$$


where $B P_{S_{j}}^{p}$ is the present value of the bid price of the transformer to be installed at the $p$-th year of the study period, $R_{S_{i}}^{p}$ is the remaining value of the uninstalled transformer, $B P_{S_{i}}$ is the transformer $S_{i}$ bid price, $r$ is the inflation rate, $N$ are the years of the transformer lifetime and $m$ are the years that the transformer was under service.

Table 2 lists the cost of four arcs of Fig. 5, calculated according to Section 4 and 6.

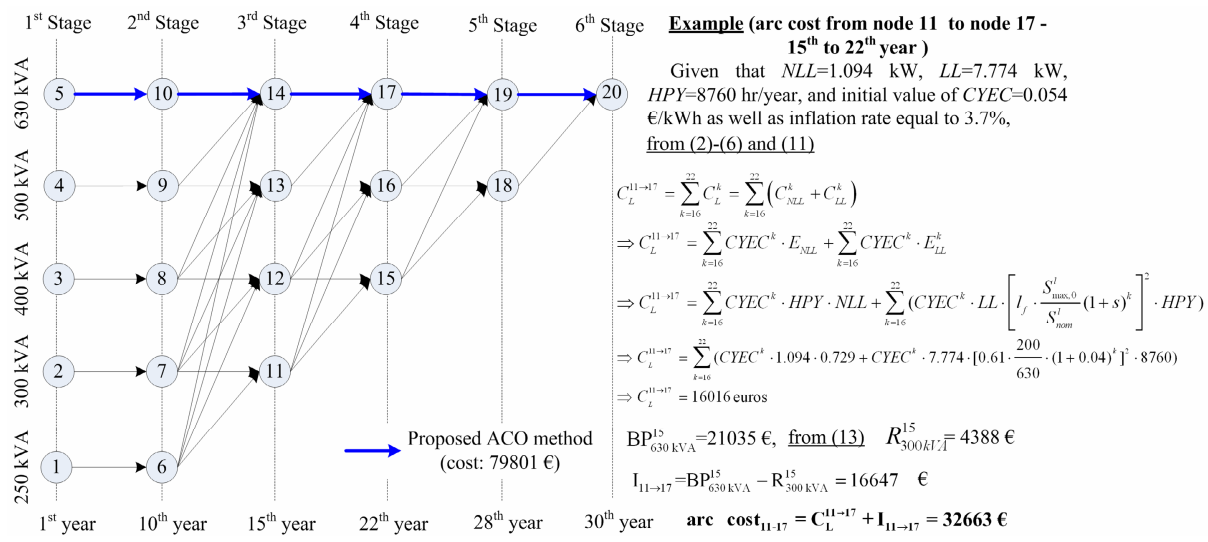

Fig. 5. The directed graph used by the proposed ACO method for the Type 5 substation load

Table 1. Technical Parameters and Thermal Withstand of the Transformers Used in the Solution of the OTS Problem

\begin{tabular}{cccc|cccccc}
\hline \multicolumn{4}{c|}{ Transformer technical parameters } & \multicolumn{5}{c}{ Transformer thermal withstand (yr) per type of substation load } \\
\hline $\begin{array}{c}\text { Size } \\
(\boldsymbol{k V A})\end{array}$ & $\begin{array}{c}\text { Bid price } \\
(\boldsymbol{\epsilon})\end{array}$ & $\begin{array}{c}\boldsymbol{N} \boldsymbol{L} \boldsymbol{L} \\
(\boldsymbol{k W})\end{array}$ & $\begin{array}{c}\boldsymbol{L} \boldsymbol{L} \\
(\boldsymbol{k W})\end{array}$ & Type 1 & Type 2 & Type 3 & Type 4 & Type 5 & Type 6 \\
\hline 160 & 5275 & 0.454 & 2.544 & 5 & 4 & 8 & 3 & 0 & 0 \\
250 & 6853 & 0.702 & 3.672 & 16 & 14 & 18 & 14 & 10 & 7 \\
300 & 6932 & 0.738 & 4.186 & 21 & 18 & 23 & 19 & 15 & 12 \\
400 & 9203 & 0.991 & 4.684 & 28 & 25 & 28 & 26 & 22 & 19 \\
500 & 10296 & 1.061 & 5.771 & 30 & 30 & 30 & 30 & 28 & 24 \\
630 & 12197 & 1.094 & 7.774 & - & - & - & - & 30 & 30 \\
\hline
\end{tabular}

Table 2. Cost of indicative arcs in the graph of Fig. 5

\begin{tabular}{llc:llc}
\hline Arc & Cost of the arc & Value $(\boldsymbol{\xi})$ & Arc & Cost of the arc & Value $(\boldsymbol{\xi})$ \\
\hline $2 \rightarrow 7$ & $C_{L}^{2 \rightarrow 7}+B P_{300}^{1 s t-y e a r}$ & 17972 & $17 \rightarrow 19$ & $C_{L}^{17 \rightarrow 19}$ & 23601 \\
$11 \rightarrow 17$ & $C_{L}^{11 \rightarrow 17}+I_{11 \rightarrow 17}$ & 32663 & $19 \rightarrow 20$ & $C_{L}^{19 \rightarrow 20}$ & 11185 \\
\hline
\end{tabular}

Fig. 5 illustrates the graph used by the proposed ACO method for the solution of the OTS problem for Type 5 substation load of Fig. 4 using the transformers of Table 1. We tested several values for each parameter, i.e. $\alpha \in\{0,0.5,1,2,5\}$, $\beta \in\{0,0.5,1,2,5\}, \rho \in\{0.1,0.3,0.5,0.7,1\}$. Table 3 includes the data of the optimal 
sizing strategies for each type of substation of Fig. 4 and Table 1 . The optimal solutions of Table 3 were obtained using $k=20, \alpha=2, \beta=0.5, \rho=0.5, Q=2.7$, $\max$ iterations $=2000$. According to Fig. 5 , the optimal sizing path yielded by the proposed method corresponds to installation of the largest rated capacity that can serve the expected load at the end of the study period. The same optimal path is selected for the rest of types of the substation loads of the considered network, corresponding to the costs listed in Table 3. This is due to the fact that transformers with rated power significantly larger than the served load operate under low load current, thus consuming less annual energy losses (and consequently having less annual energy loss cost), in comparison with transformers of rated power close to the served load.

Table 3. Results of the proposed method for the OTS problem of Fig. 4

\begin{tabular}{cccccccc}
\hline \multirow{2}{*}{ Transformer cost } & \multicolumn{7}{c}{ Substation load types } \\
\cline { 2 - 7 } & Type 1 & Type 2 & Type 3 & Type 4 & Type 5 & Type 6 & Total \\
\hline Installation cost $(€)$ & 10677 & 10296 & 10677 & 10677 & 12648 & 12648 & 177573 \\
Energy loss cost $(€)$ & 48529 & 61195 & 66372 & 56025 & 67153 & 70047 & 940822 \\
Total cost $(€)$ & 59206 & 71491 & 77049 & 66702 & 79801 & 82695 & 1118395 \\
\hline
\end{tabular}

\section{Conclusions}

In this paper, an EAS algorithm is proposed for the solution of the OTS planning problem by minimizing the overall transformer cost (i.e., the sum of the transformer purchasing cost plus the transformer energy loss cost) over the planning period, while satisfying all the problem constraints (i.e., the load to be served and the transformer thermal loading limit). The method is applied for the selection of the optimal size of the distribution transformers in a real network, comprising 16 distribution substations to serve a load over a period of 30 years. The application results show that the proposed EAS algorithm is very efficient because it always converges to the global optimum solution of the OTS problem.

Acknowledgments. This paper is part of the 03ED45 research project, implemented within the framework of the "Reinforcement Programme of Human Research Manpower" (PENED) and co-financed by National and Community Funds (25\% from the Greek Ministry of Development-General Secretariat of Research and Technology and $75 \%$ from E.U.-European Social Fund).

\section{References}

1. Chen, C.-S., Wu, T.-H.: Optimal Distribution Transformer Sizing by Dynamic Programming. Electrical Power \& Energy Systems 20, 161-167 (1998)

2. Jovanovic, D.: Planning of Optimal Location and Sizes of Distribution Transformers using Integer Programming. Electrical Power \& Energy Systems 25, 717-723 (2003)

3. Dorigo, M., Stützle, T.: Ant Colony Optimization. MIT Press, Cambridge (2004)

4. Stützle, T., Hoos, H.H.: MAX-MIN Ant System. Future Generation Computer Systems 16, 889-914 (2000) 
5. Merkle, D., Middendorf, M., Schmeck, H.: Ant Colony Optimization for Resource-constrained Project Scheduling. IEEE Transactions on Evolutionary Computation 6, 333-346 (2002)

6. Leguizamon, G., Michalewicz, Z.: A New Version of Ant System for Subset Problems. In: Proc. of the 1999 Congress on Evolutionary Computation, vol. 2, pp. 1459-1464 (1999)

7. Blum, C.: Ant Colony Optimization: Introduction and Recent Trends. Physics of Life Reviews 2, 353-373 (2005)

8. Amoiralis, E.I., Tsili, M.A., Georgilakis, P.S., Kladas, A.G.: Ant Colony Solution to Optimal Transformer Sizing Problem. In: CD Proc. of EPQU (2007)

9. IEEE Guide for Loading Mineral-Oil-Immersed Transformers, IEEE Std C57.91 (2002) 